\title{
Bunsen flame simulation by finite elements on adaptively refined, unstructured triangulations
}

\author{
Erik Burman $^{1}$, Alexandre Ern ${ }^{2,4}$ and Vincent Giovangigli ${ }^{3}$ \\ ${ }^{1}$ DMA, Ecole polytechnique fédérale de Lausanne, $\mathrm{CH}-1015$, Switzerland \\ 2 CERMICS, Ecole nationale des ponts et chaussées, 6 et 8 avenue Blaise Pascal, F-77455 Marne \\ la Vallée Cedex 2, France \\ ${ }^{3}$ CMAP, Ecole polytechnique, F-91128 Palaiseau Cedex, France \\ E-mail: erik.burman@epfl.ch,ern@cermics.enpc.fr and giovangi@cmapx.polytechnique.fr
}

Received 18 February 2003, in final form 17 October 2003

Published 5 December 2003

Online at stacks.iop.org/CTM/8/65 (DOI: 10.1088/1364-7830/8/1/004)

\begin{abstract}
We investigate laminar Bunsen flames with detailed chemistry and multicomponent transport. The governing equations are discretized by a finite element method on a sequence of adaptively refined, unstructured triangulations. The finite element method is an extension to chemically reacting flows of the streamline diffusion method, including least-squares stabilization of the pressure gradient and the low-Mach continuity equation as well as a shock capturing term designed to control species mass fraction undershoots near flame fronts. Unstructured meshes are adaptively refined based on a posteriori estimates of a user specified functional of the numerical error. These estimates are derived from the dual weighted residual method in the form of element-wise residuals weighted by coefficients depending on the solution of a linearized dual problem that accounts for convective error propagation and multicomponent chemistry couplings. Numerical results are presented to illustrate the efficiency of the proposed methodology and to study the impact of inflow velocity profiles on the structure of several hydrogen-air Bunsen flames.
\end{abstract}

\section{Introduction}

Bunsen flames are obtained experimentally by flowing a premixed fuel/oxidizer jet through a cylindrical tube. When the jet velocity exceeds the laminar flame speed, it is possible under appropriate experimental conditions to stabilize a flame of conical shape above the tube lips. Bunsen flames arise in several practical applications including household and industrial burners. Furthermore, they provide a relatively simple flow configuration on which to investigate fundamental aspects of laminar premixed combustion, including flame stabilization

4 Author to whom any correspondence should be addressed. 
mechanisms as well as chemically and hydrodynamically controlled extinction limits. The investigation of Bunsen flame structures can also yield useful information to derive new, and improve current, premixed turbulent combustion models.

Despite the extensive progress witnessed over the last few years in computer technology, Bunsen flame simulation still remains an extremely challenging task, especially when detailed chemistry modelling is taken into account. Computational difficulties stem from the disparity of time and space scales to be resolved and from the nonlinear couplings present in the governing equations. It is therefore critical to design reliable and efficient numerical methods that achieve a prescribed level of accuracy at the lowest computational cost. An important step in this direction is to derive local error indicators that solely depend on the numerical solution and the problem data. Adaptively refined meshes can then be constructed based on this information. A first strategy consists in using local estimates of the gradient and curvature of the numerical solution. Applications to combustion include [1-8]. These error indicators are the only possibility if finite difference discretizations are employed. Unfortunately, they often lack theoretical justification as soon as the problem is not single-component and diffusiondominated. This is the case in flame problems since error propagation due to convective transport and complex chemistry couplings is often dominant. Another drawback of local gradient and curvature estimates is that they do not provide a quantitative assessment of the actual numerical error.

An interesting approach to adaptive mesh generation with quantitative error control has been developed recently in the framework of finite element methods and optimal control techniques $[9,10]$. The dual weighted residual (DWR) method provides a quantitative estimate for a user specified functional of the error in terms of residuals, defined as the finite element solution re-injected into the governing equations, weighted by coefficients depending on the solution of a linearized dual problem. The dual weights account for convective error propagation and also contain information relative to complex chemistry couplings at the flame front. Moreover, the estimate can be split into element-wise error indicators to generate an adaptively refined mesh.

Application of the DWR method to combustion problems includes premixed flames in hot wall tubes and periodic slot burners with detailed chemistry $[11,12]$ as well as axisymmetric jet flames with simple chemistry [13]. In both cases, hierarchical rectangular meshes were employed. In [11,12], these meshes were directly used to discretize the governing equations with bilinear finite elements. In [13], a triangulation with no hanging nodes was constructed by dividing rectangular cells into triangles, the governing equations being then discretized with simplicial finite elements. Although hierarchical meshes offer some advantages, such as the possibility of constructing smoothing operators within multigrid methods, fully unstructured meshes are particularly attractive because of the absence of hanging nodes and the flexibility of handling complex geometries. A first step toward adaptive flame simulations on unstructured meshes was explored in [13] where these meshes were generated from error indicators obtained on structured meshes.

The goal of this paper is to show that successful error control can be achieved in the finite element simulation of laminar Bunsen flames on adaptive, fully unstructured meshes. The physical model incorporates detailed chemical kinetics and multicomponent transport algorithms, including non-diagonal molecular diffusion and thermal diffusion. While the theoretical background of the computational methodology (stabilized finite elements and the DWR method) is fairly well established, particular emphasis is laid herein on numerical aspects of error estimation and mesh generation in a fully unstructured framework. Indeed, adaptively generated, unstructured meshes do not form a nested family, and this has an impact on the evaluation of the dual weights in the error indicators and also on the way a new mesh is 
constructed from the coarser one. Results are presented for rich and lean $\mathrm{H}_{2}$-air Bunsen flames. First, we assess the error indicators derived within the present methodology using a reference solution obtained on a very fine mesh. For various error output functionals associated with temperature and chemical species, we show that reliable error control and efficient mesh adaption can be achieved. In order to evaluate the viability of the proposed methodology as a means to investigate Bunsen flame structures more systematically, we also discuss a series of simulations where the impact of injection velocities on the Bunsen flame shape is studied.

\section{Physical modelling}

This section specifies our physical model for laminar Bunsen flames, including conservation equations, complex chemistry with finite rate kinetics, multicomponent transport algorithms and boundary conditions.

\subsection{Conservation equations}

The equations governing laminar Bunsen flames express the conservation of species mass, momentum and energy. These equations are considered here in their axisymmetric and steady form. Furthermore, since the flow velocities are significantly smaller than the sound speed, we use the isobaric (or low Mach) flame approximation and split the pressure into a spatially varying hydrodynamic pressure $p$ plus a constant thermodynamic pressure $p_{0}$ in such a way that the ratio $p / p_{0}$ scales as the square of the Mach number $[14,15]$. Letting $n s$ be the number of species in the mixture, one can choose for the dependent unknowns the $(n s+4)$-vector $u$ with components

$$
u=\left(p, v_{r}, v_{z}, T, y_{1}, \ldots, y_{n s}\right),
$$

where $v_{r}$ and $v_{z}$ denote, respectively, the radial and axial velocity components, $T$ the temperature and $y_{1}, \ldots, y_{n s}$ the species mass fractions.

The governing equations can be written in the general form

$$
\mathcal{L}_{i}(u):=\mathcal{F}_{i}^{0}(u)+\frac{1}{r} \partial_{r}\left(r \mathcal{F}_{i}^{r}(u)\right)+\partial_{z}\left(\mathcal{F}_{i}^{z}(u)\right)=0, \quad 1 \leqslant i \leqslant n s+4,
$$

where $\partial_{r}$ and $\partial_{z}$ denote differentiation with respect to the radial coordinate $r$ and the axial coordinate $z$, respectively. The zero-order fluxes $\mathcal{F}^{0}(u)$ are given by

$$
\mathcal{F}^{0}(u)=\left(\begin{array}{l}
\partial_{r} v_{r}+\partial_{z} v_{z}+\frac{v_{r}}{r}+\frac{1}{\rho}\left(v_{r} \partial_{r} \rho+v_{z} \partial_{z} \rho\right) \\
\rho(v \cdot \partial) v_{r}-\frac{p}{r}-\frac{2}{3} \frac{\eta}{r}\left(\partial_{r} v_{r}+\partial_{z} v_{z}+\frac{v_{r}}{r}\right)+2 \frac{\eta}{r^{2}} v_{r} \\
\rho(v \cdot \partial) v_{z}+\rho g \\
\rho c_{p}(v \cdot \partial) T-\omega_{T} \\
\rho(v \cdot \partial) y_{i}-\omega_{i}
\end{array}\right),
$$

where $\rho$ denotes the density given by the ideal gas law

$$
\rho=\frac{p_{0} m}{R T} .
$$

In the above equations, $m$ is the mean molecular mass of the mixture, $R$ the universal gas constant, $v \cdot \partial=v_{r} \partial_{r}+v_{z} \partial_{z}$ the convective derivative, $g>0$ the gravity constant, $c_{p}$ the specific heat capacity at constant pressure of the mixture, $\omega_{T}$ the enthalpy production rate and 
$\omega_{i}$ the mass production rate of the $i$ th species. The first-order radial and axial fluxes $\mathcal{F}^{r}(u)$ and $\mathcal{F}^{z}(u)$ may be expressed as

$$
\mathcal{F}^{r}(u)=\left(\begin{array}{l}
0 \\
-2 \eta \partial_{r} v_{r}+p+\frac{2}{3} \eta\left(\partial_{r} v_{r}+\partial_{z} v_{z}+\frac{v_{r}}{r}\right) \\
-\eta\left(\partial_{r} v_{z}+\partial_{z} v_{r}\right) \\
q_{r} \\
f_{i, r}
\end{array}\right)
$$

and

$$
\mathcal{F}^{z}(u)=\left(\begin{array}{l}
0 \\
-\eta\left(\partial_{r} v_{z}+\partial_{z} v_{r}\right) \\
-2 \eta \partial_{z} v_{z}+p+\frac{2}{3} \eta\left(\partial_{r} v_{r}+\partial_{z} v_{z}+\frac{v_{r}}{r}\right) \\
q_{z} \\
f_{i, z}
\end{array}\right)
$$

where $\eta$ is the shear viscosity, $q=\left(q_{r}, q_{z}\right)$ the thermal part of the heat flux vector and $f_{i}=\left(f_{i, r}, f_{i, z}\right)$ the mass diffusion flux of the $i$ th species. The enthalpy production rate is given by

$$
\omega_{T}=-\sum_{i=1}^{n s} h_{i} \omega_{i}-\sum_{i=1}^{n s} c_{p, i}\left(f_{i, r} \partial_{r} T+f_{i, z} \partial_{z} T\right),
$$

where $h_{i}$ is the specific enthalpy of the $i$ th species and $c_{p, i}$ its specific heat capacity at constant pressure. Because of overall mass conservation, the governing equation for the last chemical species (typically a dilutant such as $\mathrm{N}_{2}$ ) is actually written as $\mathcal{L}_{n s+4}(u):=\sum_{j=1}^{n s} y_{j}-1$.

\subsection{Thermochemistry}

Thermochemical properties are computed using vectorized and highly optimized versions of the Chemkin package [16]. The species specific enthalpies and constant pressure heat capacities are evaluated as a function of the temperature using polynomial fits with tabulated coefficients. The species production rates result from a complex reaction mechanism and may be expressed as

$$
\omega_{i}=m_{i} \sum_{r=1}^{n r}\left(v_{i r}^{\mathrm{b}}-v_{i r}^{\mathrm{f}}\right)\left(K_{\mathrm{f}, r} \prod_{j=1}^{n s} c_{j}^{v_{j r}^{\mathrm{f}}}-K_{\mathrm{b}, r} \prod_{j=1}^{n s} c_{j}^{v_{j r}^{\mathrm{b}}}\right),
$$

where $m_{i}$ is the molecular mass of the $i$ th species, $n r$ the number of elementary reactions, $v_{i r}^{\mathrm{f}}$ and $v_{i r}^{\mathrm{b}}$ are, respectively, the forward and backward stoichiometric coefficients for the $i$ th species in the $r$ th elementary reaction, $K_{\mathrm{f}, r}$ and $K_{\mathrm{b}, r}$ the forward and backward reaction constants for the $r$ th elementary reaction, respectively, and $c_{j}$ the concentration of the $j$ th chemical species. The forward reaction constant $K_{\mathrm{f}, r}$ is evaluated as a function of the temperature using a modified Arrhenius expression. The backward constant is recovered from the relation $K_{\mathrm{b}, r}=K_{\mathrm{f}, r} / K_{\mathrm{e}, r}$, where $K_{\mathrm{e}, r}$ is the equilibrium constant given by thermodynamics. In our simulations, we consider a reaction mechanism involving $n s=9$ species $\mathrm{H}_{2}, \mathrm{O}_{2}, \mathrm{H}_{2} \mathrm{O}, \mathrm{H}, \mathrm{O}$, $\mathrm{OH}, \mathrm{HO}_{2}, \mathrm{H}_{2} \mathrm{O}_{2}$ and $\mathrm{N}_{2}$ participating in $n r=19$ elementary reactions [17]. 


\subsection{Multicomponent transport algorithms}

From the kinetic theory of dilute polyatomic gas mixtures and the first-order Enskog-Chapman expansion, the fluxes expressing multicomponent transport of species mass, momentum and energy take the form

$$
\begin{aligned}
& f_{i}=-\sum_{j=1}^{n s} \rho y_{i} D_{i j}\left(\partial x_{j}+x_{j} \tilde{\chi}_{j} \partial \log T\right), \quad 1 \leqslant i \leqslant n s \\
& S=-2 \eta \varepsilon+\frac{2}{3} \eta \operatorname{tr}(\varepsilon) I \\
& q=-\lambda \partial T+R T \sum_{j=1}^{n s} \frac{\tilde{\chi}_{j}}{m_{j}} f_{j}
\end{aligned}
$$

where $x_{j}$ is the mole fraction of the $j$ th species, $\partial=\left(\partial_{r}, \partial_{z}\right)$ the gradient operator, $D=\left(D_{i j}\right)_{1 \leqslant i, j \leqslant n s}$ the multicomponent diffusion matrix, $\tilde{\chi}=\left(\tilde{\chi}_{j}\right)_{1 \leqslant j \leqslant n s}$ the rescaled thermal diffusion ratios, $\varepsilon$ the strain tensor given by

$$
\varepsilon=\left(\begin{array}{ccc}
\partial_{r} v_{r} & 0 & \frac{1}{2}\left(\partial_{r} v_{z}+\partial_{z} v_{r}\right) \\
0 & \frac{v_{r}}{r} & 0 \\
\frac{1}{2}\left(\partial_{r} v_{z}+\partial_{z} v_{r}\right) & 0 & \partial_{z} v_{z}
\end{array}\right)
$$

and $\lambda$ the thermal conductivity. The multicomponent diffusion matrix $D$ is symmetric positive semidefinite with kernel spanned by the vector $\left(y_{1}, \ldots, y_{n s}\right)$ and the rescaled thermal diffusion ratios are such that $\sum_{j=1}^{n s} x_{j} \tilde{\chi}_{j}=0$. As a result, the species diffusion fluxes are compatible with the overall mass conservation constraint

$$
\sum_{j=1}^{n s} f_{j}=0 .
$$

Non-diagonal molecular diffusion and thermal diffusion are accounted for in the multicomponent fluxes (9) because these effects often have a sizeable impact on laminar and turbulent $\mathrm{H}_{2}$-air flame structures [18-20].

The multicomponent transport coefficients $\rho D, \tilde{\chi}, \lambda$ and $\eta$ depend on the temperature and the species mass fractions. To evaluate these coefficients accurately and cost-effectively, convergent iterative methods based on the mathematical structure of the transport linear systems given by the kinetic theory are employed [21]. The transport coefficients $\tilde{\chi}, \lambda$ and $\eta$ are computed as detailed in [18]. Furthermore, the species mass diffusion fluxes are evaluated from the positive definite version of the Stefan-Maxwell-Boltzmann equations without the intermediate calculation of the multicomponent diffusion matrix $D$. Let $\Delta$ be the matrix with coefficients $\Delta_{i j}=-x_{i} x_{j} / \mathcal{D}_{i j}$, for $1 \leqslant i, j \leqslant n s$ and $i \neq j$, and $\Delta_{i i}=-\sum_{j \neq i} \Delta_{i j}$ where $\mathcal{D}_{i j}$ is the binary diffusion coefficient for species pair $(i, j)$. Let $Y=\left(y_{1}, \ldots, y_{n s}\right)$ be the mass fraction vector and let $d=\left(d_{1}, \ldots, d_{n s}\right)$ be the vector with components $d_{i}=\partial x_{i}+x_{i} \tilde{\chi}_{i} \partial \log T$. The species mass diffusion fluxes are then given by $f_{i}=\rho y_{i} \zeta_{i}$ where $\zeta \in \mathbb{R}^{n s}$ solves

$$
(\Delta+Y \otimes Y) \zeta=-d .
$$

The constraint (11) is satisfied by construction. The above multicomponent transport algorithms are implemented within the EGlib package [22]. 


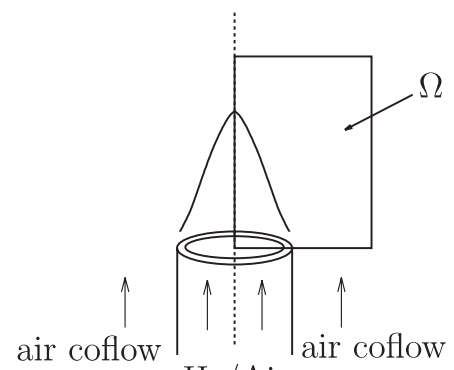

$\mathrm{H}_{2} / \mathrm{Air}$

Figure 1. Physical configuration for the axisymmetric Bunsen flame problem (not to scale)

\subsection{Boundary conditions}

The governing equations discussed in the previous sections are posed on a computational domain $\Omega$ which in our simulations will be a rectangular domain $\left[0, L_{r}\right] \times\left[0, L_{z}\right]$. The boundary $\partial \Omega$ is split into its four sides numbered clockwise, with $\partial \Omega_{1}$ denoting the axis of symmetry $(r=0), \partial \Omega_{2}$ the outflow boundary $\left(z=L_{z}\right), \partial \Omega_{3}$ the far field $\left(r=L_{r}\right)$ and $\partial \Omega_{4}$ the inflow boundary $(z=0)$. The physical configuration is depicted in figure 1 .

The governing equations are closed by the following boundary conditions:

- axis of symmetry

$$
\partial_{r} p=0, \quad v_{r}=0, \quad \partial_{r} v_{z}=0, \quad \partial_{r} T=0, \quad \partial_{r} y_{i}=0,
$$

- outflow

$$
p=0, \quad v_{r}=0, \quad \partial_{z} v_{z}=0, \quad \partial_{z} T=0, \quad \partial_{z} y_{i}=0,
$$

- far field

$$
\partial_{r} p=0, \quad v_{r}=0, \quad \partial_{r} v_{z}=0, \quad \partial_{r} T=0, \quad \partial_{r} y_{i}=0,
$$

- inflow

$$
v_{r}=0, \quad v_{z}=v^{d}(r), \quad T=T^{d}, \quad \rho^{d}(r) v^{d}(r)\left(y_{i}-y_{i}^{d}(r)\right)+f_{i, z}=0,
$$

where the superscript $d$ indicates prescribed values.

\section{Numerical methods}

This section describes the numerical methods considered in this paper: the finite element discretization of the governing equations, the a posteriori error estimate based on the DWR method and the algorithmic aspects related to adaptive grid generation.

\subsection{Finite element discretization}

Let $\mathcal{T}$ be a triangulation (with no hanging nodes) of the computational domain $\Omega$. Let $P_{\mathrm{c}}^{1}$ be the conforming finite element space with linear trial functions given by

$$
P_{\mathrm{c}}^{1}=\left\{\phi \in C^{0}(\bar{\Omega}), \forall K \in \mathcal{T}, \phi_{\mid K} \in \mathbb{P}^{1}\right\},
$$


where $\mathbb{P}^{1}$ is the set of polynomials of degree $\leqslant 1$. Set also $P_{\mathrm{c}, 0}^{1}=\left\{\phi \in P_{\mathrm{c}}^{1}, \phi_{\mid \partial \Omega}=0\right\}$ and for $1 \leqslant l \leqslant 4, P_{\mathrm{c}, l}^{1}=\left\{\phi \in P_{\mathrm{c}}^{1}, \phi_{\mid \partial \Omega_{l}}=0\right\}$.

We seek a discrete solution $u_{*}=\left(p_{*}, v_{r *}, v_{z_{*}}, T_{*}, y_{1 *}, \ldots, y_{n s *}\right) \in u_{*}^{d}+\mathbb{V}$ where $u_{*}^{d}$ accounts for non-homogeneous Dirichlet boundary conditions (velocity and temperature at inflow) and

$$
\mathbb{V}=P_{\mathrm{c}, 2}^{1} \times P_{\mathrm{c}, 0}^{1} \times P_{\mathrm{c}, 4}^{1} \times P_{\mathrm{c}, 4}^{1} \times\left(P_{\mathrm{c}}^{1}\right)^{n s}
$$

In the Galerkin formulation, the approximate solution $u_{*}$ is required to satisfy the discrete equations

$$
\forall \phi=\left(\phi_{1}, \ldots, \phi_{n s+4}\right) \in \mathbb{V}, \quad a\left(u_{*} ; \phi\right)=0
$$

where

$$
\begin{aligned}
a\left(u_{*} ; \phi\right)= & \sum_{i=1}^{n s+4} \int_{\Omega} \mathcal{F}_{i}^{0}\left(u_{*}\right) \cdot \phi_{i} r \mathrm{~d} r \mathrm{~d} z-\sum_{i=1}^{n s+4} \int_{\Omega} \mathcal{F}_{i}^{r}\left(u_{*}\right) \cdot \partial_{r} \phi_{i} r \mathrm{~d} r \mathrm{~d} z \\
& \quad-\sum_{i=1}^{n s+4} \int_{\Omega} \mathcal{F}_{i}^{z}\left(u_{*}\right) \cdot \partial_{z} \phi_{i} r \mathrm{~d} r \mathrm{~d} z+\sum_{i=1}^{n s} \int_{\partial \Omega_{4}} \rho^{d} v^{d}\left(y_{i}-y_{i}^{d}\right) \phi_{4+i} r \mathrm{~d} l .
\end{aligned}
$$

The form $a$ is nonlinear in $u_{*}$ but linear in $\phi$.

Some additional terms must be added to the form $a$ to stabilize the Galerkin finite element formulation (19). The stabilization is designed to match the following targets:

- least-squares control of streamline derivatives for velocity components, temperature and species mass fractions [23-25];

- least-squares control of hydrodynamic pressure gradient and of continuity equation [26, 27];

- high-order shock capturing term to control spurious oscillations near sharp fronts [28].

To write the stabilization terms, it is convenient to introduce the following numerical parameters

$$
\begin{aligned}
\beta=\left(\beta_{r}, \beta_{z}\right)= & \left(\rho_{*} v_{r *}, \rho_{*} v_{z *}\right), \quad \beta^{\perp}=\left(\beta_{z},-\beta_{r}\right), \quad|\beta|=\left(\beta_{r}^{2}+\beta_{z}^{2}\right)^{1 / 2}, \\
& \delta_{\mathrm{sd}}=\frac{1}{2}\left(\frac{\delta}{h^{2}}+\frac{|\beta|}{h}\right)^{-1}, \quad \delta=10^{-5}, \\
& \delta_{\mathrm{cd}}=0.4 h^{1 / 2} \delta_{\mathrm{sd}}, \\
& \delta_{\mathrm{div}}=4 h|\beta|,
\end{aligned}
$$

where $\rho_{*}$ is the density evaluated from the approximate solution $u_{*}$. All the numerical parameters in (21) are computed locally in each triangle $K \in \mathcal{T}$ at the centre of gravity. Because the Prandtl number and the species Lewis numbers are not significantly different from unity, the same reference diffusion coefficient $\delta$ is used for momentum, energy and species mass. This approximation improves significantly the conditioning of the Jacobian matrices that are considered when solving the discrete equations while keeping the correct asymptotic behaviour for the streamline diffusion coefficient $\delta_{\mathrm{sd}}$. 
The stabilization form may be expressed locally over a triangle $K \in \mathcal{T}$ as

$$
\begin{aligned}
b_{K}\left(u_{*} ; \phi\right)= & \int_{K} \sum_{i=4}^{n s+3} \delta_{\text {sd }} \mathcal{L}_{i}\left(u_{*}\right)\left(\beta \cdot \partial \phi_{i}\right) r \mathrm{~d} r \mathrm{~d} z \\
& +\int_{K} \delta_{\text {sd }}\left(\mathcal{L}_{2}\left(u_{*}\right)\left(\beta \cdot \partial \phi_{2}+\partial_{r} \phi_{1}\right)+\mathcal{L}_{3}\left(u_{*}\right)\left(\beta \cdot \partial \phi_{3}+\partial_{z} \phi_{1}\right)\right) r \mathrm{~d} r \mathrm{~d} z \\
& +\int_{K} \delta_{\mathrm{div}} \mathcal{L}_{1}\left(u_{*}\right)\left(\partial_{r} \phi_{2}+\frac{\phi_{2}}{r}+\partial_{z} \phi_{3}+\frac{v_{r *}}{T_{*}} \partial_{r} \phi_{4}+\frac{v_{z *}}{T_{*}} \partial_{z} \phi_{4}\right) r \mathrm{~d} r \mathrm{~d} z \\
& +\int_{K} \delta_{\mathrm{cd}}\left(\left(\beta^{\perp} \cdot \partial v_{r *}\right)\left(\beta^{\perp} \cdot \partial \phi_{2}\right)+\left(\beta^{\perp} \cdot \partial v_{z *}\right)\left(\beta^{\perp} \cdot \partial \phi_{3}\right)\right) r \mathrm{~d} r \mathrm{~d} z \\
& +\int_{K} \delta_{\mathrm{cd}}\left(\left(\beta^{\perp} \cdot \partial T_{*}\right)\left(\beta^{\perp} \cdot \partial \phi_{4}\right)+\sum_{i=1}^{n s-1}\left(\beta^{\perp} \cdot \partial y_{i *}\right)\left(\beta^{\perp} \cdot \partial \phi_{4+i}\right)\right) r \mathrm{~d} r \mathrm{~d} z .
\end{aligned}
$$

The first two lines on the right-hand side of (22), which correspond to the streamline diffusion method and the least-squares control of the hydrodynamic pressure gradient, have been considered in a wide range of applications involving convection-dominated flows or pressurevelocity instabilities due to the same order of discretization for both variables. The third line yields a least-squares control of the low-Mach continuity equation and is introduced in this work as an extension of the symmetric stabilization term usually considered for incompressible flows. The last two lines are high-order, shock capturing terms whose function is to help preserve mass fraction positivity in the vicinity of sharp fronts. Note that only crosswind diffusion terms are considered.

Since the residuals of the governing equations $\mathcal{L}_{i}\left(u_{*}\right)$ are evaluated element-wise and the approximate solution $u_{*}$ is linear in each triangle, second derivatives due to diffusion fluxes vanish identically. A further simplification is introduced by neglecting the spatial derivatives of multicomponent transport coefficients in the local residuals. We thus consider local stabilization terms $\tilde{b}_{K}\left(u_{*} ; \phi\right)$ obtained from (22) upon substituting the residuals $\mathcal{L}_{i}\left(u_{*}\right)$ by the local residuals $\mathcal{R}_{i}\left(u_{*}\right)$ given by

$$
\begin{aligned}
& \mathcal{R}_{1}\left(u_{*}\right)=\partial_{r} v_{r}+\partial_{z} v_{z}+\frac{v_{r}}{r}+\frac{1}{\rho}\left(v_{r} \partial_{r} \rho+v_{z} \partial_{z} \rho\right), \\
& \mathcal{R}_{2}\left(u_{*}\right)=\rho(v \cdot \partial) v_{r}+\partial_{r} p-\frac{4}{3} \frac{\eta}{r}\left(\partial_{r} v_{r}-\frac{v_{r}}{r}\right), \\
& \mathcal{R}_{3}\left(u_{*}\right)=\rho(v \cdot \partial) v_{z}+\partial_{z} p+\rho g-\frac{\eta}{r}\left(\partial_{r} v_{z}+\frac{1}{3} \partial_{z} v_{r}\right), \\
& \mathcal{R}_{4}\left(u_{*}\right)=\rho c_{p}(v \cdot \partial) T+\frac{q_{r}}{r}-\omega_{T}, \\
& \mathcal{R}_{4+i}\left(u_{*}\right)=\rho(v \cdot \partial) y_{i}+\frac{f_{i, r}}{r}-\omega_{i},
\end{aligned}
$$

the right-hand side being evaluated using the approximate solution $u_{*}$. The stabilized FEM formulation then consists in seeking $u_{*} \in u_{*}^{0}+\mathbb{V}$ such that

$$
\forall \phi \in \mathbb{V}, \quad a\left(u_{*} ; \phi\right)+\sum_{K \in \mathcal{T}} \tilde{b}_{K}\left(u_{*} ; \phi\right)=0 .
$$

\subsection{A posteriori error estimation}

Let $e=\left(e_{1}, \ldots, e_{n s+4}\right)$ be the error with components $e_{i}=u_{i}-u_{* i}, 1 \leqslant i \leqslant n s+4$. Given a function $\psi \in L^{2}(\Omega)^{n s+4}$, the error output measure

$$
\mathcal{J}(e)=\int_{\Omega}(\psi \cdot e) r \mathrm{~d} r \mathrm{~d} z
$$


can be estimated using the DWR method $[9,10]$. Recall that the estimate takes the form

$$
\mathcal{J}(e) \leqslant \sum_{K \in \mathcal{T}} \eta_{K}
$$

with local error indicators $\eta_{K}=\sum_{i=1}^{n s+4} \varsigma_{K, i} w_{K, i}$. The finite element residuals $\varsigma_{K, i}$ are given by

$$
\varsigma_{K, i}=h_{K}\left\|\mathcal{L}_{i}\left(u_{*}\right)\right\|_{K}+\frac{1}{2} h_{K}^{1 / 2}\left\|\left[\left(\mathcal{F}_{i}^{r}, \mathcal{F}_{i}^{z}\right) \cdot n\right]\right\|_{\partial K},
$$

where $h_{K}$ is the diameter of $K,\|\cdot\|_{K}$ the $L^{2}(K)$-norm, $\partial K$ the boundary of $K, n$ its outward normal and $\left[\left(\mathcal{F}_{i}^{r}, \mathcal{F}_{i}^{z}\right) \cdot n\right]$ the jump of the normal diffusion fluxes across $\partial K$ in the direction of $n$. The weights $w_{K, i}$ are defined from the solution, $\xi$, of a linearized dual problem (see $[9,10,12,13]$ for more details).

The evaluation of the finite element residuals is relatively straightforward and is briefly described for completeness. We first notice that neglecting spatial derivatives of multicomponent transport coefficients, the first term on the right-hand side of (27) can be written as $h_{K}\left\|\mathcal{R}_{i}\left(u_{*}\right)\right\|_{K}$. Using one-point nodal quadrature at the centre of gravity, $G(K)$, of $K$, we obtain $|K|^{-1 / 2}\left\|\mathcal{R}_{i}\left(u_{*}\right)\right\|_{K}=r_{\mid G(K)}^{1 / 2}\left|\mathcal{R}_{i}\left(u_{*}\right)\right|_{G(K)}+\mathcal{O}\left(h_{K}\right)$ provided the second derivatives of the residual can be bounded uniformly in $h_{K}$. Proceeding similarly for the second term in (27), yields

$$
\varsigma_{K, i} \simeq h_{K}^{2} r_{\mid G(K)}^{1 / 2}\left|\mathcal{R}_{i}\left(u_{*}\right)\right|_{G(K)}+\frac{1}{2} \sum_{e \in \partial K} h_{K} r_{\mid G(K)}^{1 / 2}\left|\left[\left(\mathcal{F}_{i}^{r}, \mathcal{F}_{i}^{z}\right) \cdot n\right]\left(m_{e}\right)\right|,
$$

where the measure of $K$ and its edges have been evaluated as $h_{K}^{2}$ and $h_{K}$, respectively, and where $m_{e}$ is the midpoint of edge $e$.

On unstructured meshes, a specific computational aspect is the evaluation of the weights $\omega_{K, i}$. As in previous work, a discrete solution to a dual problem linearized at the approximate solution $u_{*}$ is sought, i.e. we seek $\xi_{*} \in \mathbb{V}$ such that

$$
\forall \phi \in \mathbb{V}, \quad a^{\prime}\left(u_{*} ; \phi, \xi_{*}\right)+\sum_{K \in \mathcal{T}} \tilde{b}_{K}^{\prime}\left(u_{*} ; \phi, \xi_{*}\right)=\mathcal{J}(\phi),
$$

where $a^{\prime}\left(u_{*} ; \phi, \cdot\right)$ is the Gateaux derivative of $a$ in the direction of $\phi$ evaluated at $u_{*}$ and a similar notation is used for $\tilde{b}_{K}^{\prime}$. The weights are then evaluated as $c_{\text {int }} h_{K}\left\|D_{K}^{2} \xi_{*}\right\|_{K}$ where $c_{\text {int }}$ is an interpolation constant and $D_{K}^{2} \xi_{*}$ a local approximation to the second derivatives of the exact dual solution $\xi$. The interpolation constant is estimated by 1 , which is generally an upper bound. To approximate second derivatives on unstructured meshes, let $i_{*}$ be the interpolation operator

$$
i_{*}: \phi \in P^{0} \mapsto i_{*} \phi=\sum_{j=1}^{n n o} \phi_{j} N_{j} \in P_{\mathrm{c}}^{1},
$$

where $P^{0}$ is the space of piecewise constant functions and

$$
\phi_{j}=\frac{\sum_{K \in \mathcal{T}_{j}}|K| \phi_{\mid K}}{\sum_{K \in \mathcal{T}_{j}}|K|}
$$

and $\mathcal{T}_{j}$ is the set of triangles in $\mathcal{T}$ containing vertex $j$ and $N_{j}$ the Lagrange nodal basis function associated with this vertex. Second derivatives of $\xi$ are then approximated in the spirit of the Zienkewicz-Zhu estimator [29] by differentiating the local $P^{1}$ reconstruction of the gradient of $\xi_{*}$, i.e. $\partial\left(i_{*} \partial \xi_{*}\right)$. Because of the axisymmetric setting, we consider

$$
D_{K}^{2} \xi_{* i}=\max \left(\left|\delta_{K}^{r r} \xi_{* i}\right|,\left|\delta_{K}^{z z} \xi_{* i}\right|, \frac{1}{2}\left|\delta_{K}^{r z} \xi_{* i}+\delta_{K}^{z r} \xi_{* i}\right|, \frac{1}{r_{\mid G(K)}}\left|\partial_{r} \xi_{* i \mid K}\right|\right),
$$


where $\delta_{K}^{\alpha \beta} \xi_{* i}=\partial_{\alpha}\left(s_{*} \partial_{\beta} \xi_{* i}\right)_{\mid K}$ for $\alpha, \beta \in\{r, z\}$. This yields

$$
w_{K, i} \simeq h_{K}^{2} r_{G(K)}^{1 / 2} D_{K}^{2} \xi_{* i}
$$

where, as before, $|K|^{1 / 2}$ has been evaluated as $h_{K}$. The numerical analysis of (33) relies on the interpolation property

$$
\sum_{K \in \mathcal{T}} h_{K}^{-2}\left\|\partial \phi_{*}-i_{*} \partial \phi_{*}\right\|_{K}^{2} \leqslant c\|\phi\|_{H^{2}(\Omega)}^{2},
$$

where $c$ denotes a mesh-independent constant. The above inequality is valid provided $\phi$ belongs to the Sobolev space $H^{2}(\Omega),\left\|\partial\left(\phi-\phi_{*}\right)\right\|_{K}$ is first-order in $h_{K}$ and the mesh is locally quasi-uniform (see [30] for more details). To conclude, we use the local inverse inequality

$$
\left\|\partial\left(i_{*} \partial \phi_{*}\right)\right\|_{K} \leqslant h_{K}^{-1}\left\|\partial \phi_{*}-i_{*} \partial \phi_{*}\right\|_{K},
$$

valid since $\partial \phi_{*}$ is constant on $K$ and provided the mesh is shape-regular. Assuming the dual solutions $\xi$ and $\xi_{*}$ verify the above assumptions, this yields $\left\|\partial\left(i_{*} \partial \xi_{*}\right)\right\|_{L^{2}(\Omega)} \leqslant c\|\xi\|_{H^{2}(\Omega)}$, showing that the reconstructed second derivatives are upper-bounded by the $H^{2}(\Omega)$-norm of the exact dual solution. The numerical experiments presented in the next section confirm the viability of these approximations for error control in Bunsen flame simulations.

\subsection{Adaptive mesh refinement}

The meshes considered in this work are fully unstructured, quasi-Delaunay triangulations generated using the advancing front method. The mesh generator is described in [31] and uses two input files: the first specifies the trace of the mesh on the boundary $\partial \Omega$ and is referred to as the boundary mesh file. The second specifies the desired mesh size around a cloud of points located inside $\Omega$ and is referred to as the background mesh file.

The core of the adaptive mesh refinement procedure is to create boundary and background mesh files from the information provided by the local error indicators $\eta_{K}$. The adaptive algorithm involves three steps:

(i) compute desired mesh sizes

$$
h_{K}^{\prime}=f\left(\eta_{K}\right) h_{K}
$$

where for instance, $f\left(\eta_{K}\right)=\frac{1}{2}$ if $\eta_{K} \geqslant$ TOL and $f\left(\eta_{K}\right)=1$ otherwise. The function $f$ can also take values larger than 1 for very small $\eta_{K}$ to allow for local mesh coarsening. An error balancing method is employed in which the tolerance TOL is evaluated as

$$
\mathrm{TOL}=\frac{1}{2} \frac{1}{n t} \sum_{K \in \mathcal{T}} \eta_{K},
$$

where $n t$ is the number of triangles in the current mesh;

(ii) the boundary mesh file is determined directly from the quantities $h_{K}^{\prime}$ using the triangles having at least an edge located on $\partial \Omega$;

(iii) the background mesh file is also directly generated from the provisional $h_{K}^{\prime}$. To avoid excessive specifications in this file, a layering algorithm is employed in which the desired mesh size of a given triangle is written to the background mesh file only if its value is sufficiently different from that of the neighbouring elements. The fluctuation test is for instance $\left|\log \left(h_{K_{1}}^{\prime} / h_{K_{2}}^{\prime}\right)\right| \geqslant \log \gamma$ with parameter $\gamma=1.5$. 


\subsection{Numerical solution methodology}

The discrete equations (24) are conveniently recast into a system of nonlinear equations upon introducing the decomposition

$$
u_{* i}=\sum_{j=1}^{n n o} U_{i, j} N_{j}, \quad 1 \leqslant i \leqslant n s+4,
$$

where nno is the number of mesh vertices (including boundary ones). For $1 \leqslant i \leqslant n s+4$ and $1 \leqslant j \leqslant n n o$, let $\phi_{i, j} \in \mathbb{V}$ be the test function with components $\left(\delta_{i k} N_{j}\right)_{1 \leqslant k \leqslant n s+4}$ where $\delta_{i k}$ is the Kronecker symbol. The discrete unknowns form a vector $U \in \mathbb{R}^{(n s+4) * n n o}$ with components $U_{i, j}$ satisfying the nonlinear discrete equations

$$
F_{i, j}(U)=a\left(u_{*} ; \phi_{i, j}\right)+\sum_{K \in \mathcal{T}} \tilde{b}_{K}\left(u_{*} ; \phi_{i, j}\right)=0 .
$$

The residuals $F_{i, j}(U)$ are evaluated using numerical quadratures with three Gauss points located at midpoints of triangle edges.

The residual equations (38) are written in vector form $F(U)=0$ and an approximate solution is obtained using a damped Newton's method. Given an initial guess $U^{0}$, the damped Newton's method yields the sequence of iterates

$$
\partial_{U} F\left(U^{k}\right)\left(\bar{U}^{k+1}-U^{k}\right)=-\sigma^{k} F\left(U^{k}\right),
$$

where $\partial_{U} F\left(U^{k}\right)$ is the Jacobian matrix of $F$ evaluated at $U^{k}, \bar{U}^{k+1}$ the provisional Newton iterate and $\sigma^{k}$ the damping parameter. The Newton iterate $U^{k+1}$ is then evaluated from $\bar{U}^{k+1}$ using a projection on the cone $\Lambda$ of positive mass fractions. Convergence of Newton's method is achieved when the norm of the update vector $U^{k+1}-U^{k}$ is brought below a prescribed tolerance, typically $10^{-4}$. The projection on $\Lambda$ is compatible with the convergence of Newton's method if the projection error is smaller than the prescribed tolerance. This is indeed the case in our computations owing to the shock capturing terms used to stabilize the Galerkin formulation.

At each Newton step (39), the Jacobian matrix is assembled from perturbed residual evaluations and the linear system is solved approximately using a preconditioned Krylov iterative solver, typically BiCGStab with Gauss-Seidel preconditioner blocked at the node level. The efficiency of the preconditioner is enhanced by an appropriate renumbering of the mesh nodes according to the flow streamlines. Convergence is achieved when the relative linear residual has been brought below a prescribed tolerance, typically $10^{-7}$. Because the source terms present in the local residuals (23) have a significant impact on the conditioning of the Jacobian matrix, it is more efficient to consider on coarse meshes a first-order streamline diffusion method in which the local species residuals only contain the convective-diffusive contribution. On fine meshes where convective-diffusive-reactive effects are adequately resolved at the flame front, the high-order expressions (23) can be used. This point will be further discussed below.

Once convergence has been achieved on a given mesh, the local error indicators $\eta_{K}$ are used to assess the numerical error and, if necessary, to construct a new, adaptively refined, unstructured mesh. While the finite element residuals $\varsigma_{K, i}$ are readily evaluated, the computation of the weights $w_{K, i}$ requires the solution of the following linearized dual problem: seek $X \in \mathbb{R}^{(n s+4) * n n o}$ such that

$$
\partial_{U} F^{\mathrm{T}} \cdot X=\Psi
$$

where $\partial_{U} F^{\mathrm{T}}$ denotes the transpose of the last Jacobian used in the damped Newton's method and where the right-hand side $\Psi \in \mathbb{R}^{(n s+4) * n n o}$ has components $\Psi_{i, j}=\mathcal{J}\left(\phi_{i, j}\right)$ for $1 \leqslant i \leqslant n s+4$ and $1 \leqslant j \leqslant n n o$. The nodal values of the dual solution $\xi_{*}$ are then directly recovered from 
the components of the vector $X$ and are used in (31). Note that the computational effort to solve (40) is much lower than that required to obtain the discrete solution $U$.

\section{Numerical results}

In this section, we discuss our numerical results. We consider rich and lean $\mathrm{H}_{2}$-air Bunsen flames. The premixed fresh gas mixture is injected through a tube with inner radius $r_{\mathrm{i}}=2 \mathrm{~mm}$ and outer radius $r_{\mathrm{o}}=2.5 \mathrm{~mm}$. Both the burner flow and the air coflow are of plug type with exponential boundary layer

$$
v^{\mathrm{d}}(r)= \begin{cases}v_{\mathrm{b}}\left(1-\exp \left(\frac{r-r_{\mathrm{i}}}{\delta}\right)\right) & r \leqslant r_{\mathrm{i}}, \\ 0 & r_{\mathrm{i}} \leqslant r \leqslant r_{\mathrm{o}}, \\ v_{\mathrm{c}}\left(1-\exp \left(\frac{r_{\mathrm{o}}-r}{\delta}\right)\right) & r_{\mathrm{o}} \leqslant r\end{cases}
$$

and gradient parameter $\delta=0.5 \mathrm{~mm}$. The burner lips, the fresh mixture flow and the air coflow are kept at a temperature of $T^{\mathrm{d}}=298 \mathrm{~K}$. In section 4.1, numerical errors corresponding to various output functionals are assessed on a sequence of adaptively refined, unstructured triangulations by comparing them with estimates obtained from a reference flame on a very fine mesh. To illustrate the fact that the present methodology can be used to investigate more systematically laminar flame structures, we study in section 4.2 the influence of injection velocity profiles on rich Bunsen flame shapes.

\subsection{Evaluation of the adaptive methodology}

We first evaluate the adaptive methodology on rich Bunsen flames. The fresh $\mathrm{H}_{2}$-air mixture contains $75 \%$ hydrogen and $5.2 \%$ oxygen in mole fraction. This yields an equivalence ratio of 7.2, for which the laminar flame speed is $19.5 \mathrm{~cm} \mathrm{~s}^{-1}$ [19]. Injection velocities in (41) are given by $v_{\mathrm{b}}=v_{\mathrm{c}}=120 \mathrm{~cm} \mathrm{~s}^{-1}$. Figure 2 presents isotherms and mole fraction isocontours for species $\mathrm{H}, \mathrm{OH}$ and $\mathrm{HO}_{2}$. The computational domain, as in all subsequent simulations, has dimensions $L_{r}=1.5$ and $L_{z}=25 \mathrm{~cm}$. We observe a cold dark zone circumscribed by the conically shaped premixed flame front. Although the temperature peaks at the cone tip located on the symmetry axis, its value remains nearly constant along the flame front, in agreement with the Clavin and Williams theory since the overall Lewis number for oxygen is
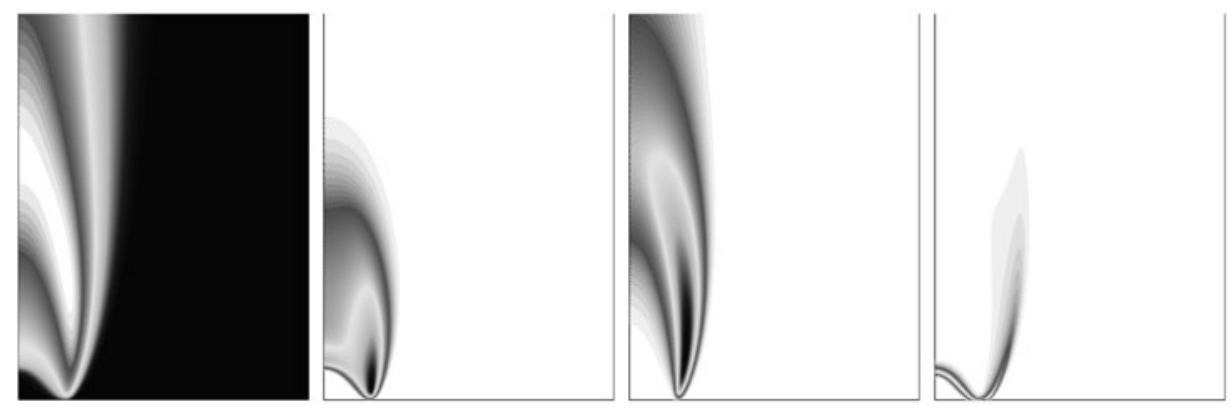

Figure 2. From left to right: isotherms and mole fraction isocontours for species $\mathrm{H}, \mathrm{OH}$ and $\mathrm{HO}_{2}$; peak values are, respectively, $2038 \mathrm{~K}, 1.74 \times 10^{-2}, 1.07 \times 10^{-2}$ and $4.39 \times 10^{-4}$; the plotting domain is $[0,1.5] \times[0,2]$ in $\mathrm{cm}$; rich Bunsen flame. 

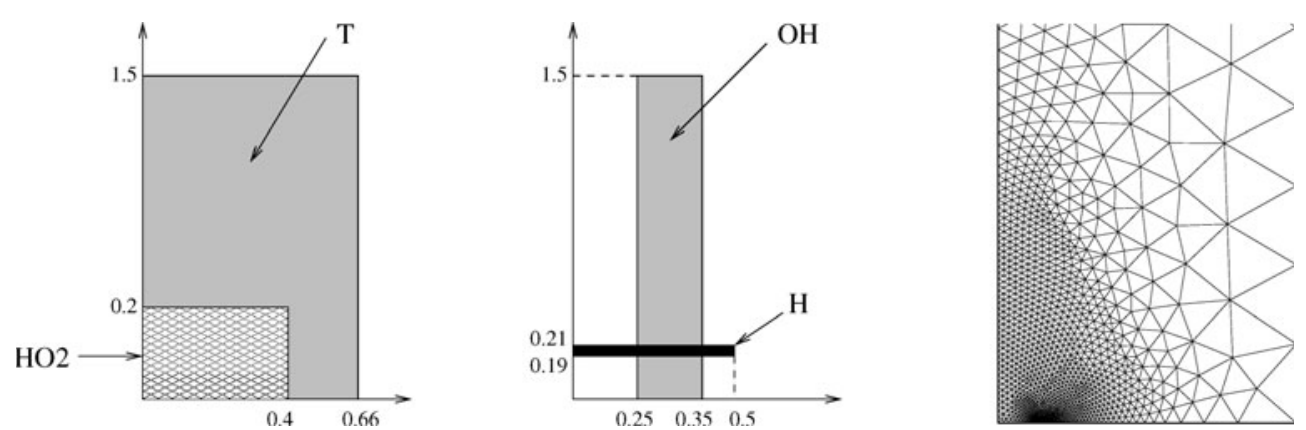

Figure 3. Illustration (not to scale) of the four numerical strategies for adaptive error control and initial coarse triangulation.

about unity [32]. The flame length, defined from the point on the symmetry axis where the temperature reaches its maximum, is $1.26 \mathrm{~cm}$ and the flame lift-off, defined as the lowest $z$ for which the temperature is above $1000 \mathrm{~K}$, is $0.11 \mathrm{~mm}$. Most of the hydrogen is not consumed at the premixed flame front, but diffuses radially to create an outer diffusion flame where it burns with the oxygen supplied by the air coflow. This phenomenon is clearly visible in the $\mathrm{H}$ and $\mathrm{OH}$ mole fraction isocontours.

To control the average error on a given solution component and over a specified region of the flow, we consider error output functionals of the form

$$
\mathcal{J}_{m, \omega}(e)=\frac{1}{|\omega|} \int_{\omega} e_{m} r \mathrm{~d} r \mathrm{~d} z,
$$

where $m$ denotes a solution component with $1 \leqslant m \leqslant n s+4, \omega$ a sub-domain of the computational domain $\Omega$ and $|\omega|$ its measure. The sub-domain $\omega$ typically covers the flame front or follows a line along which experimental measurements are made. We investigate the four choices illustrated in figure 3 for (42). We propose to control errors on either the temperature over sub-domain $[0,0.66] \times[0,1.5]$, or the $\mathrm{H}$ mole fraction over sub-domain $[0,0.5] \times[0.19,0.21]$, or the $\mathrm{OH}$ mole fraction over sub-domain $[0.25,0.35] \times[0,1.5]$, or the $\mathrm{HO}_{2}$ mole fraction over sub-domain $[0,0.4] \times[0,0.2]$ (units in centimetres). In all cases, the adaptive procedure is started with an initial coarse unstructured triangulation containing 1599 nodes and 2954 elements (see figure 3). This mesh is constructed with very little a priori knowledge on the flame structure. In the boundary mesh file, we simply set $h$ to $0.05 \mathrm{~mm}$ at the burner lip, to $0.3 \mathrm{~mm}$ at the lower left corner and to $4 \mathrm{~mm}$ at the upper and right boundaries. The background mesh file further imposes a desired mesh size of $h=0.4 \mathrm{~mm}$ at points $(0.2,0.8)$ and $(0.3,1) \mathrm{cm}$ inside the computational domain. For each of the error output functionals depicted in figure 3 , four levels of adaptive refinement are performed. To assess the error indicators, a reference flame obtained on a grid with 61468 nodes is used.

Figure 4 presents the unstructured meshes generated after two steps of the adaptive algorithm. We observe that local mesh refinement occurs at quite different locations depending on the targeted solution component. For error control on the temperature over the whole flame, mesh refinement occurs above the burner lips, inside the premixed flame cone, and along the trailing diffusion flame. The adaptive mesh based on error control for the $\mathrm{OH}$ mole fraction has a comparable number of nodes because this radical is very sensitive to the temperature profile. The adaptive mesh for $\mathrm{H}$ error control is substantially different from the previous two triangulations and also from the shape of the $\mathrm{H}$ mole fraction isocontours. Indeed, because of multicomponent chemistry couplings, the accuracy achieved for the $\mathrm{H}$ mole fraction strongly depends on the resolution of various radical profiles. This information can only be captured 

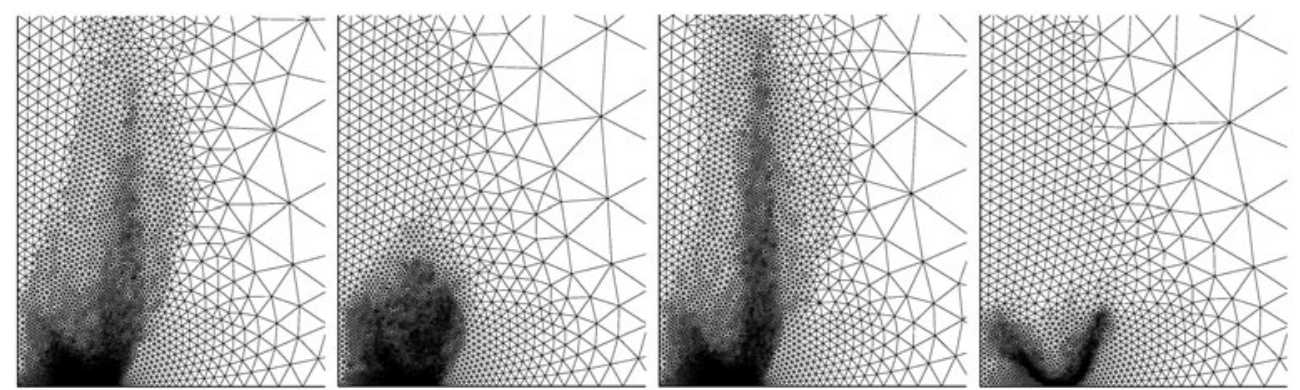

Figure 4. Unstructured meshes generated after the second adaptive refinement step; from left to right: temperature, $\mathrm{H}, \mathrm{OH}$ and $\mathrm{HO}_{2}$ error output functionals; rich Bunsen flame.
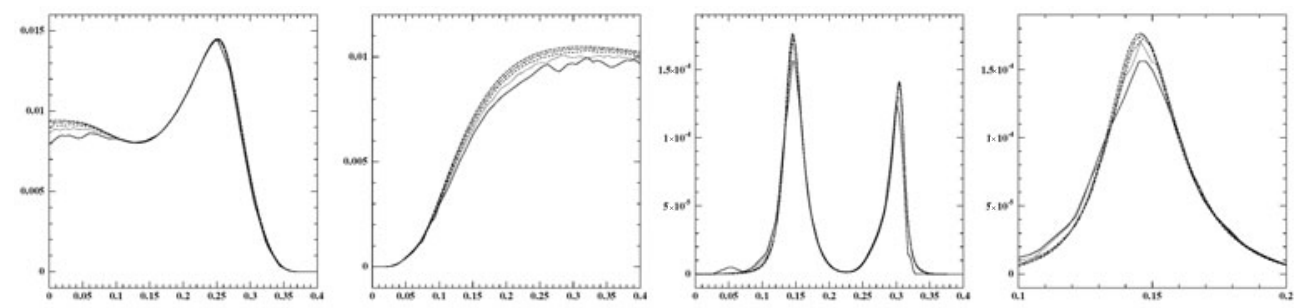

Figure 5. Solution profiles along selected lines as computed during the adaptive process; from left to right: $\mathrm{H}$ mole fraction at $z=2 \mathrm{~mm}, \mathrm{OH}$ mole fraction at $r=3 \mathrm{~mm}, \mathrm{HO}_{2}$ mole fraction at $z=0.5 \mathrm{~mm}$ and a zoom of the $\mathrm{HO}_{2}$ profile; rich Bunsen flame.

Table 1. Adaptive error control for the temperature: error indicators (in K) given by the DWR method and errors estimated using a reference solution; rich Bunsen flame.

\begin{tabular}{lrllllll}
\hline Level & Nodes & Indicator & Order & $\begin{array}{l}\text { Estimated } \\
\mathcal{J} \text {-error }\end{array}$ & Order & $\begin{array}{l}\text { Effectivity } \\
\text { index }\end{array}$ & $\begin{array}{l}\text { Estimated } \\
L^{1} \text {-error }\end{array}$ \\
\hline 0 & 1599 & $1.22 \times 10^{2}$ & - & $1.39 \times 10^{1}$ & - & 8.8 & $2.04 \times 10^{1}$ \\
1 & 2826 & $5.55 \times 10^{1}$ & 1.39 & $9.79 \times 10^{0}$ & 0.61 & 5.7 & $1.35 \times 10^{1}$ \\
2 & 5948 & $2.76 \times 10^{1}$ & 0.94 & $5.94 \times 10^{0}$ & 0.67 & 4.6 & $8.31 \times 10^{0}$ \\
3 & 13987 & $1.40 \times 10^{1}$ & 0.79 & $3.09 \times 10^{0}$ & 0.77 & 4.5 & $4.10 \times 10^{0}$ \\
4 & 34577 & $7.60 \times 10^{0}$ & 0.67 & $1.06 \times 10^{0}$ & 1.20 & 7.2 & $1.33 \times 10^{0}$ \\
\hline
\end{tabular}

by solving a dual problem. The adaptive mesh based on $\mathrm{HO}_{2}$ error control concentrates most of its degrees of freedom above the burner lips and in the upstream part of both the premixed flame cone and the trailing diffusion flame. Further downstream, the $\mathrm{HO}_{2}$ radical is consumed and a coarser mesh can be used. Figure 5 presents solution profiles along selected lines as computed during the adaptive process. For each radical, we compare the profiles obtained using the corresponding error functional. The improvement in capturing the radical profiles is clearly visible, especially for $\mathrm{HO}_{2}$ where adequate resolution is achieved only at the fourth adaptive refinement step.

Numerical results for temperature error control are presented in table 1 . The first column indicates the iteration number $l$ in the adaptive algorithm, the second the number of nodes $n n o_{l}$ of the corresponding unstructured triangulation, the third the error indicator $\eta_{l}$ evaluated using the DWR method described in section 3.2, the fourth the convergence order of $\eta_{l}$, the fifth the estimated $\mathcal{J}$-error $\mathcal{J}\left(e_{l}\right)$ evaluated using the reference solution, the sixth the convergence 
order of $\mathcal{J}\left(e_{l}\right)$, the seventh the effectivity index defined by the ratio $\eta_{l} / \mathcal{J}\left(e_{l}\right)$ and the eighth the estimated $L^{1}$-error $\mathcal{J}\left(\left|e_{l}\right|\right)$. For a quantity $\alpha_{l}$ depending on the adaptive refinement step $l$ (either the error indicator $\eta_{l}$ or the estimated error $\mathcal{J}\left(e_{l}\right)$ ), the convergence order $\sigma(\alpha)$ is evaluated from the expression

$$
\sigma(\alpha)=\frac{\log \left(\alpha_{l-1} / \alpha_{l}\right)}{\log \left(\text { nno }_{l} / \text { nno }_{l-1}\right)} .
$$

We observe from table 1 that owing to the error balancing method, the number of nodes increases smoothly from one mesh to the next finer one. The ratio of the number of nodes on consecutive triangulations generally ranges between two and three. Furthermore, on all adaptive meshes, the estimated $\mathcal{J}$-error is very close to the estimated $L^{1}$-error showing that very few algebraic cancellations occur in the temperature error over the flame. The convergence order of the estimated errors ranges between 0.6 and 0.8 up to level 3 . This is in agreement with theoretical results for linear finite elements stabilized by the streamline diffusion method on globally refined meshes where a convergence order $\sigma=\frac{3}{4}$ is expected for the $L^{2}$-error. Furthermore, on levels $1-3$, the effectivity index is fairly constant, ranging from 4 to 6 . On the coarsest mesh, the dual solution is not resolved well enough to capture the correct error behaviour. On the finest mesh, the effectivity index is also larger, but no definitive conclusion can be drawn since the $\mathcal{J}$-error is computed using a fine mesh solution instead of the exact solution. To sum up, the results reported in table 1 indicate that reliable error control can be achieved for the temperature on adaptive unstructured grids.

Numerical results for $\mathrm{OH}$ error control are reported in table 2. Most of the conclusions drawn for the temperature carry over. In particular, convergence orders are compatible with theoretical estimates, and the effectivity index remains fairly constant on levels $1-3$. The value is, however, larger than that associated with temperature by a factor of 2 . This can be attributed to the more oscillatory behaviour of the dual solution. Numerical results for $\mathrm{H}$ and $\mathrm{HO}_{2}$ error control are summarized in table 3. Both cases are more difficult to tackle numerically since on the one hand the control domain for the $\mathrm{H}$ error is very thin (see figure 3 ) and on the

Table 2. Adaptive error control for $\mathrm{OH}$ : error indicators given by the DWR method and errors estimated using a reference solution; rich Bunsen flame.

\begin{tabular}{lrllllll}
\hline Level & Nodes & Indicator & Order & $\begin{array}{l}\text { Estimated } \\
\mathcal{J} \text {-error }\end{array}$ & Order & $\begin{array}{l}\text { Effectivity } \\
\text { index }\end{array}$ & $\begin{array}{l}\text { Estimated } \\
L^{1} \text {-error }\end{array}$ \\
\hline 0 & 1599 & $1.91 \times 10^{-3}$ & - & $1.28 \times 10^{-4}$ & - & 14.9 & $1.32 \times 10^{-4}$ \\
1 & 2800 & $8.82 \times 10^{-4}$ & 1.37 & $8.10 \times 10^{-5}$ & 0.81 & 10.8 & $8.44 \times 10^{-5}$ \\
2 & 6056 & $4.37 \times 10^{-4}$ & 0.91 & $4.78 \times 10^{-5}$ & 0.68 & 9.2 & $4.99 \times 10^{-5}$ \\
3 & 14844 & $2.34 \times 10^{-4}$ & 0.70 & $2.44 \times 10^{-5}$ & 0.78 & 9.6 & $2.62 \times 10^{-5}$ \\
4 & 37540 & $1.33 \times 10^{-4}$ & 0.60 & $1.07 \times 10^{-5}$ & 0.92 & 13.3 & $1.07 \times 10^{-5}$ \\
\hline
\end{tabular}

Table 3. Adaptive error control for $\mathrm{H}$ and $\mathrm{HO}_{2}$ : error indicators given by the DWR method and convergence orders; rich Bunsen flame.

\begin{tabular}{lrllllll}
\hline & \multicolumn{3}{c}{$\mathrm{H}$} & & \multicolumn{3}{c}{$\mathrm{HO}_{2}$} \\
\cline { 2 - 3 } \cline { 6 - 7 } Level & Nodes & Indicator & Order & & Nodes & Indicator & Order \\
\hline 0 & 1599 & $9.35 \times 10^{-5}$ & - & & 1599 & $1.30 \times 10^{-4}$ & - \\
1 & 2511 & $4.66 \times 10^{-5}$ & 1.54 & & 1891 & $3.79 \times 10^{-5}$ & 7.37 \\
2 & 4687 & $2.33 \times 10^{-5}$ & 1.11 & & 2771 & $1.25 \times 10^{-5}$ & 2.90 \\
3 & 10390 & $1.23 \times 10^{-5}$ & 0.80 & & 4996 & $4.66 \times 10^{-6}$ & 1.67 \\
4 & 25387 & $6.73 \times 10^{-6}$ & 0.68 & & 11638 & $2.14 \times 10^{-6}$ & 0.92 \\
\hline
\end{tabular}


other hand the $\mathrm{HO}_{2}$ radical concentrates in a very narrow region. Our numerical results reflect these difficulties. Convergence orders close to those observed for the temperature and $\mathrm{OH}$ are only reached after two or three steps in the adaptive algorithm. On coarser meshes, the $\mathrm{H}$ and $\mathrm{HO}_{2}$ profiles are not resolved well enough, as already noticed from figure 5. Effectivity indices for $\mathrm{H}$ error control on the finer meshes range between 10 and 15 and are thus close to those obtained for $\mathrm{OH}$. For $\mathrm{HO}_{2}$ error control, the estimated $\mathcal{J}$ - and $L^{1}$-errors using the reference solution differ significantly, indicating that algebraic cancellations occur so that the error indicator strongly overestimates the actual error. However, the effectivity index remains relatively constant (and close to 100) on all levels, showing that despite these cancellations, the error indicator and the estimated error have similar orders of convergence.

As discussed before, the first-order streamline diffusion method has been used in the above simulations since on coarse meshes, the inclusion of the species source terms deteriorates the conditioning of the Jacobian matrix. We have verified numerically that starting from the converged solution with first-order streamline diffusion on the finer meshes, Newton's method exhibits a smooth convergence behaviour towards the high-order solution. The a posteriori error estimates corresponding to both strategies are actually very close, confirming the adequate resolution of the inner flame structure. For instance, the error estimate on the temperature is $1.46 \mathrm{~K}$ for the converged solution with high-order streamline diffusion on the third adaptively refined mesh. This value should be compared with the value of $1.40 \mathrm{~K}$ obtained with the first-order method.

Finally, we briefly investigate the suitability of the present methodology to simulate lean Bunsen flames. The fresh $\mathrm{H}_{2}$-air mixture contains $20 \%$ hydrogen and $16.8 \%$ oxygen in mole fraction. This yields an equivalence ratio of 0.595 , for which the laminar flame speed is $86.4 \mathrm{~cm} \mathrm{~s}^{-1}$ [19]. Injection velocities in (41) are set to $v_{\mathrm{b}}=v_{\mathrm{c}}=160 \mathrm{~cm} \mathrm{~s}^{-1}$. Figure 6 presents isotherms and mole fraction isocontours for species $\mathrm{H}, \mathrm{OH}$ and $\mathrm{HO}_{2}$. The maximum temperature is not reached on the axis, a phenomenon attributable to the overall Lewis number of hydrogen, which is lower than one [32]. Mole fractions for $\mathrm{H}$ and $\mathrm{OH}$ peak slightly upstream of the premixed flame front. The $\mathrm{HO}_{2}$ radical is present along the premixed flame front up to the cone vertex, but also above the tube lip where outward diffusion of $\mathrm{H}_{2}$ results in the formation of a small diffusion flame. The flame structure has been computed using four adaptive refinement steps starting with an initial mesh containing 2025 nodes. We consider temperature error control over the same domain as for the rich flames. Figure 7, which presents the initial and the following three adaptive meshes, illustrates how the resolution of the flame front is steadily improved. Error indicators along with convergence orders are reported in table 4 . The results are very similar to those obtained for rich flames.
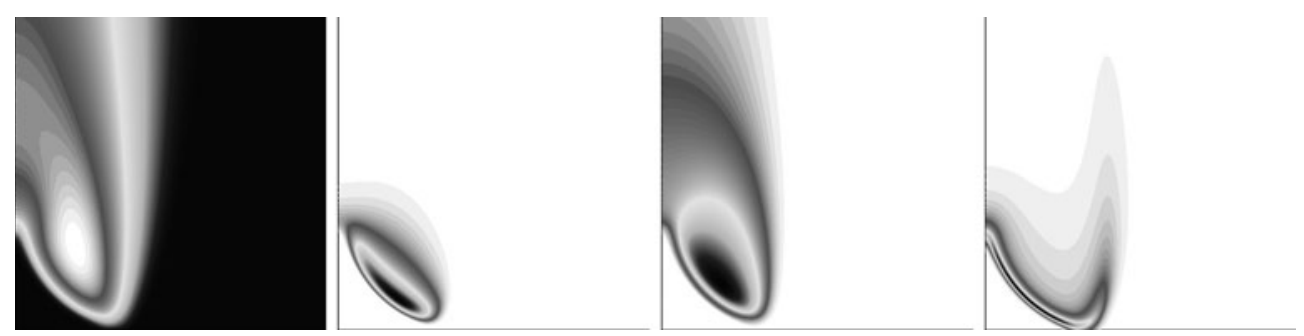

Figure 6. From left to right: isotherms and mole fraction isocontours for species $\mathrm{H}, \mathrm{OH}$ and $\mathrm{HO}_{2}$; peak values are, respectively, $1718 \mathrm{~K}, 9.86 \times 10^{-3}, 6.95 \times 10^{-2}$ and $4.10 \times 10^{-4}$; the plotting domain is $[0,0.7] \times[0,0.7] \mathrm{cm}$; lean Bunsen flame. 


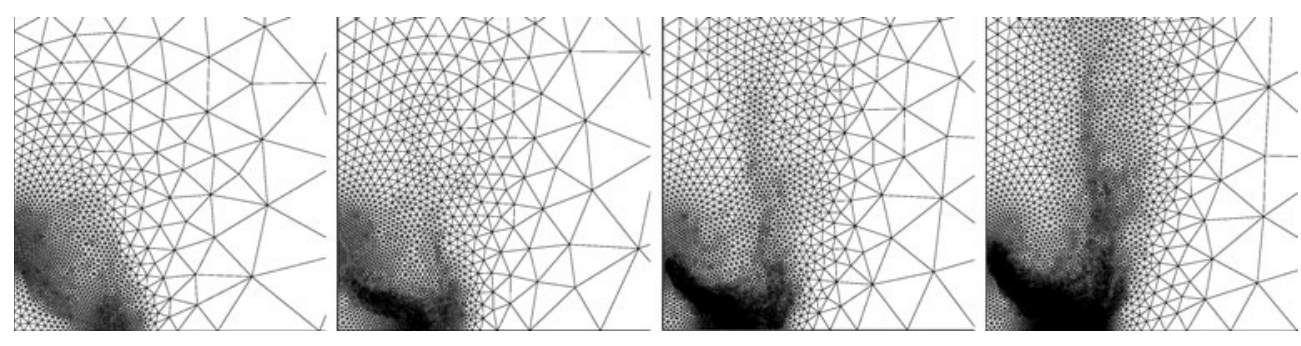

Figure 7. Adaptively refined, unstructured meshes based on temperature error control; lean Bunsen flame.

Table 4. Number of nodes, temperature error indicators (in K), and convergence orders; lean Bunsen flame.

\begin{tabular}{llllll}
\hline Level & 0 & 1 & 2 & 3 & 4 \\
\hline Nodes & 2025 & 3075 & 6207 & 13966 & 35216 \\
Indicator & $2.03 \times 10^{1}$ & $1.17 \times 10^{1}$ & $7.12 \times 10^{0}$ & $3.60 \times 10^{0}$ & $2.11 \times 10^{0}$ \\
Order & - & 1.22 & 0.75 & 0.83 & 0.58 \\
\hline
\end{tabular}

\subsection{Impact of injection velocities on flame shapes}

In this section, we investigate the impact of injection velocities on rich Bunsen flame structures. Numerical simulations are performed on the second, temperature-based, adaptively refined mesh containing 5948 nodes. On this mesh, the error in the temperature is estimated to be $27.6 \mathrm{~K}$, i.e. approximately $1 \%$ of the peak temperature. Two experiments are considered. In the first one, both the burner and the coflow velocities are varied according to

$$
v_{\mathrm{b}}=v_{\mathrm{c}} \in\{30,60,90,120,150,180,210\} \mathrm{cm} \mathrm{s}^{-1} .
$$

Figure 8 presents profiles for various solution components along selected lines. We observe important modifications in the flame length and the species profiles as the injection velocities are increased. For injection velocities lower than $90 \mathrm{~cm} \mathrm{~s}^{-1}$, the premixed flame front is located very near the inflow boundary $z=0$ and the $\mathrm{H}_{2}$ mole fraction does not reach the value supplied by the fresh gas mixture. This phenomenon, due to upstream diffusion of intermediate species, is accounted for owing to the inflow boundary condition (16). The peak value reached by radicals such as $\mathrm{H}, \mathrm{O}$ and $\mathrm{HO}_{2}$ is also significantly modified by the injection velocities (peak values increase with $v_{\mathrm{b}}$ and $v_{\mathrm{c}}$ ). The most important changes are observed for the $\mathrm{H}$ profile along the symmetry line where changes by a factor of 4 are obtained.

In our second experiment, we consider the following values for the injection velocities

$$
v_{\mathrm{b}}=120 \mathrm{~cm} \mathrm{~s}^{-1}, \quad v_{\mathrm{c}} \in\{30,60,90,120\} \mathrm{cm} \mathrm{s}^{-1} .
$$

Figure 9 presents profiles for various solution components along selected lines. We observe that the temperature along the symmetry axis is not significantly modified. However, as the coflow velocity is decreased, the flame front extends more outwards radially and the radical profiles are shifted accordingly. Peak values of $\mathrm{OH}$ and $\mathrm{HO}_{2}$ profiles decrease when $v_{\mathrm{c}}$ is diminished but the changes are less important than in the previous experiment. All the flame structures presented in this section have been obtained using a straightforward continuation procedure. Four to five steady Newton iterations are sufficient to achieve convergence from one flame structure to the next. 

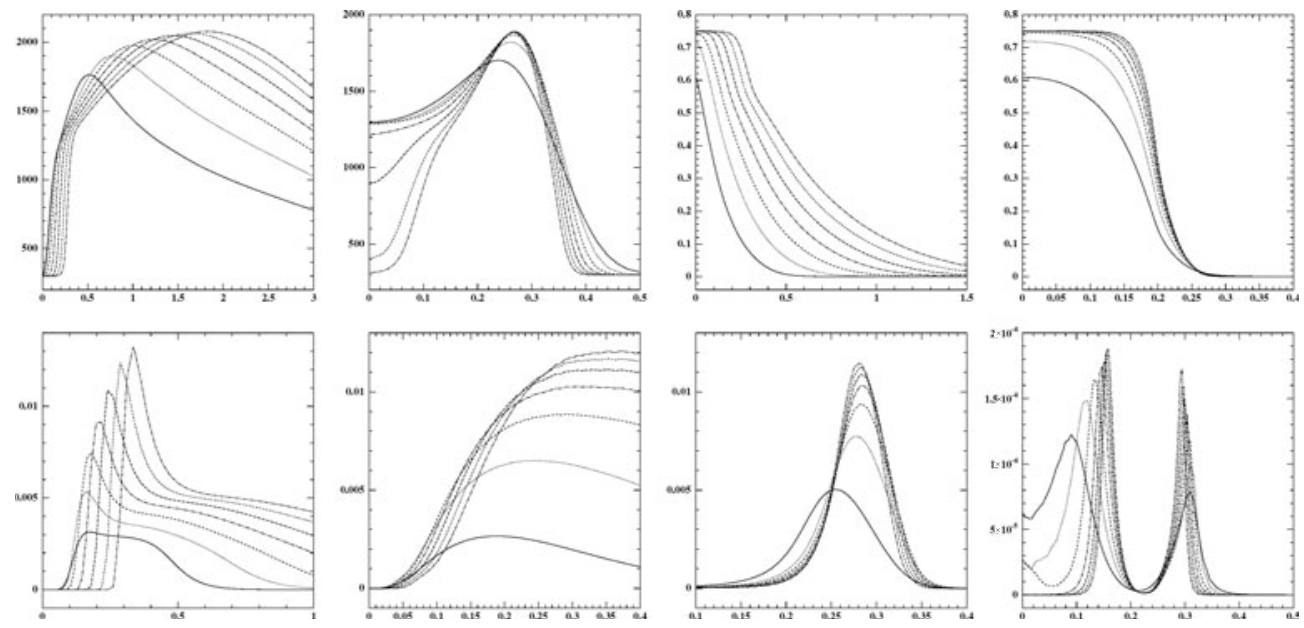

Figure 8. Profiles for various solution components along selected lines; burner and coflow velocities are varied according to (44); from left to right (top): $T$ along symmetry line, $T$ at $r=2 \mathrm{~mm}, \mathrm{H}_{2}$ mole fraction along symmetry line, $\mathrm{H}_{2}$ mole fraction at $z=0$; from left to right (bottom): $\mathrm{H}$ mole fraction along symmetry line, $\mathrm{OH}$ mole fraction at $r=3 \mathrm{~mm}, \mathrm{OH}$ mole fraction at $z=2 \mathrm{~mm}$ and $\mathrm{HO}_{2}$ at $z=0.5 \mathrm{~mm}$.
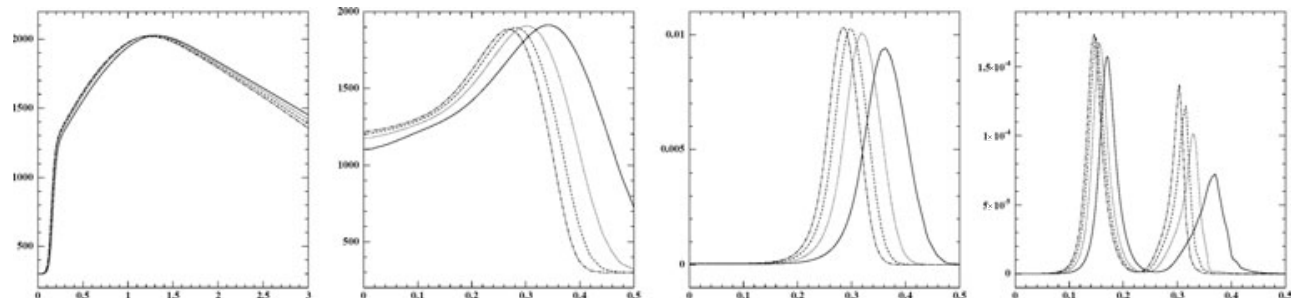

Figure 9. Profiles for various solution components along selected lines; burner and coflow velocities are varied according to (45); from left to right: $T$ along symmetry line, $T$ at $r=2 \mathrm{~mm}$, OH mole fraction at $z=2 \mathrm{~mm}$ and $\mathrm{HO}_{2}$ at $z=0.5 \mathrm{~mm}$.

\section{Conclusions}

In this paper, we have evaluated the numerical efficiency of stabilized finite element methods on adaptively refined, unstructured meshes to simulate $\mathrm{H}_{2}$-air Bunsen flames with detailed chemistry and multicomponent transport. We have considered rich and lean flame structures with varying injection velocities. Our numerical results show that the streamline diffusion method alone cannot cope efficiently with the nonlinear instabilities present in reactive fronts. Appropriate modifications include shock capturing terms ensuring positivity of the species mass fractions and first-order modifications of the method on coarse meshes.

Furthermore, our numerical results show that the DWR method provides an efficient tool to drive numerical solutions towards convergence by controlling user specified functionals of the numerical error. For an accurate resolution of the overall flame structure, temperature and $\mathrm{OH}$ error control work well. The associated error indicators exhibit convergence orders close to values expected theoretically, and the effectivity indices confirm the sharpness of the estimates. When specific regions or chemical species are targeted within the flame, computational savings can be achieved by using the appropriate error output functional. For $\mathrm{H}$ and $\mathrm{HO}_{2}$ error control, 
the error indicators are found to overestimate the actual error on the coarser meshes. The estimates become sharper after a couple of adaptive refinements for H error control, but not for $\mathrm{HO}_{2}$ because of the high resolution needed to capture the primal and dual profiles. Nevertheless, as the unstructured triangulations are adaptively refined, the cells concentrate in the appropriate regions and the species profiles are eventually captured.

In view of their recent developments, it seems interesting to investigate in future work the suitability of discontinuous Galerkin (DG) and continuous interior-penalty (CIP) methods for flame simulations. DG methods offer the advantage of enhanced stability, but at the price of a significant increase in the degrees of freedom for the discrete solution and also in the number of non-zero entries per row of the Jacobian matrix. In CIP methods, the continuous Galerkin method is stabilized by an interior-penalty operator that controls the jumps of the gradient of the discrete solution across element boundaries. For a theoretical analysis with application to convection-diffusion and incompressible flow problems, we refer to [30,33,34]. An important advantage of CIP methods over DG methods is the lower number of degrees of freedom. Furthermore, the stabilization of the CIP method is compatible with mass matrix lumping, a decisive advantage with respect to the standard streamline diffusion approach if stiff reactive source terms are present in the equations.

\section{Acknowledgment}

This paper was finished while the second author was a guest at the Bernoulli Center of the Ecole polytechnique fédérale de Lausanne, the support of which is gratefully acknowledged.

\section{References}

[1] Smooke M D 1982 Solution of burner-stabilized premixed laminar flames by boundary value methods J. Comput. Phys. 48 72-105

[2] Giovangigli V and Smooke M D 1989 Adaptive continuation algorithms with application to combustion problems Appl. Numer. Math. 5 305-31

[3] Coelho P J and Pereira J C F 1993 Calculation of a confined axisymmetric laminar diffusion flame using a local grid refinement technique Combust. Sci. Technol. 92 243-64

[4] de Lange H C and de Goey L P H 1994 Numerical modelling in a locally refined grid Int. J. Numer. Mech. Eng. 37 497-515

[5] Mallens R M M, de Lange H C, van de Ven C H J and de Goey L P H 1995 Modeling of confined and unconfined laminar premixed flames on slit and tube burners Combust. Sci. Technol. 107 387-401

[6] Somers L T and de Goey L P H 1995 A numerical study of a premixed flame on a slit burner Combust. Sci. Technol. 108 121-32

[7] Bennett B A V and Smooke M D 1998 Local rectangular refinement with application to axisymmetric laminar flames Combust. Theory Modelling 2 221-58

[8] Day M S and Bell J B 2000 Numerical simulation of laminar reacting flows with complex chemistry Combust. Theory Modelling 4 535-56

[9] Becker R and Rannacher R 1996 A feed-back approach to error control in finite element methods: basic analysis and examples East-West J. Numer. Math. 4 237-64

[10] Becker R and Rannacher R 2001 An optimal control approach to a posteriori error estimation in finite element methods Acta Numerica ed A Iserles (Cambridge: Cambridge University Press)

[11] Braack M 1998 An adaptive finite element method for reactive flow problems PhD Dissertation Heidelberg University

[12] Becker R, Braack M and Rannacher R 1999 Numerical simulation of laminar flames at low Mach number by adaptive finite elements Combust. Theory Modelling 3 503-34

[13] Burman E, Ern A and Giovangigli V 2003 Adaptive finite element methods for low Mach, steady, laminar combustion J. Comput. Phys. 188 472-92

[14] Williams F A 1985 Combustion Theory 2nd edn (Reading, MA: Addison-Welsey)

[15] Giovangigli V 1999 Multicomponent Flow Modeling (Boston, MA: Birkhäuser) 
[16] Giovangigli V and Darabiha N 1988 Vector computers and complex chemistry combustion Proc. Conf. Mathematical Modeling in Combustion and Related Topics ed C Brauner and C Schmidt-Lainé (NATO Adv. Sci. Inst. Ser. E) vol 140, pp 491-503

[17] Miller J, Mitchell R, Smooke M and Kee R J 1982 Toward a comprehensive chemical kinetic mechanism for the oxidation of acetylene: comparison of model predictions with results from flame and shock tube experiments Proc. Combust. Inst. 19 181-96

[18] Ern A and Giovangigli V 1998 Thermal diffusion effects in hydrogen-air and methane-air flames Combust. Theory Modelling 2 349-72

[19] Ern A and Giovangigli V 1999 Impact of detailed multicomponent transport on planar and counterflow hydrogen/air and methane/air flames Combust. Sci. Technol. 149 157-81

[20] de Charentenay J and Ern A 2002 Multicomponent transport impact on turbulent premixed $\mathrm{H}_{2} / \mathrm{O}_{2}$ flames Combust. Theory Modelling 6 439-62

[21] Ern A and Giovangigli V 1994 Multicomponent Transport Algorithms (Lecture Notes in Physics vol M 24) New Series Monographs (Heidelberg: Springer)

[22] Ern A and Giovangigli V 1996 Eglib server with user's manual, http://www.cmap.polytechnique.fr/www.eglib

[23] Brooks A N and Hughes T J R 1982 Streamline upwind/Petrov-Galerkin formulations for convective dominated flows with particular emphasis on the incompressible Navier-Stokes equations Comput. Methods Appl. Mech. Eng. 32 199-259

[24] Johnson C, Nävert U and Pitkäranta J 1984 Finite element methods for linear hyperbolic equations Comput. Methods Appl. Mech. Eng. 45 285-312

[25] Johnson C 1987 Numerical Solution of Partial Differential Equations by the Finite Element Method (Cambridge: Cambridge University Press)

[26] Franca L P and Frey S L 1992 Stabilized finite element methods: II. The incompressible Navier-Stokes equations Comput. Methods Appl. Mech. Eng. 99 209-33

[27] Tobiska L and Verfürth R 1996 Analysis of a streamline diffusion finite element method for the Stokes and Navier-Stokes equations SIAM J. Numer. Anal. 33 107-27

[28] Johnson C, Schatz A and Wahlbin L 1987 Crosswind smear and pointwise error in streamline diffusion finite element methods Math. Comput. 49 25-38

[29] Zienkewicz O C and Zhu J Z 1987 A simple error estimator and adaptive procedure for practical engineering analysis Int. J. Numer. Methods Eng. 24 337-57

[30] Burman E and Hansbo P 2003 Edge stabilization for Galerkin approximations of convection-diffusion-reaction problems Comput. Methods Appl. Mech. Eng. at press

[31] Rebay S 1993 Efficient unstructured mesh generation by means of Delaunay triangulation and Bowyer-Watson algorithm J. Comput. Phys. 106 125-38

[32] Clavin P and Williams F 1982 Effects of molecular diffusion and of thermal expansion on the structure and dynamics of premixed flames in turbulent flows of large scale and low intensity J. Fluid Mech. 116 251-82

[33] Burman E and Ern A 2003 Stabilized Galerkin approximation of convection-diffusion-reaction equations: discrete maximum principle and convergence Math. Comput. submitted (Internal Report 02.2003, EPFL, Switzerland)

[34] Burman E and Hansbo P 2003 Edge stabilization for the generalized Stokes problem: a continuous interiorpenalty method Math. Mod. Numer. Anal. submitted 